\title{
On the $p$-Version of the Schwab-Borchardt Mean II
}

\author{
Edward Neuman \\ Mathematical Research Institute, 144 Hawthorn Hollow, Carbondale, IL 62903, USA \\ Correspondence should be addressed to Edward Neuman; edneuman76@gmail.com
}

Received 3 November 2014; Accepted 9 March 2015

Academic Editor: Charles E. Chidume

Copyright (C) 2015 Edward Neuman. This is an open access article distributed under the Creative Commons Attribution License, which permits unrestricted use, distribution, and reproduction in any medium, provided the original work is properly cited.

\begin{abstract}
This paper deals with the $p$-version of the Schwab-Borchardt mean. Lower and upper bounds for this mean, expressed in terms of the weighted geometric and arithmetic means of its variables, are obtained. Applications to four bivariate means, introduced earlier by the author of this paper, are included.
\end{abstract}

\section{Introduction}

In the past few years, there is a renewed interest in investigations of bivariate means. In this paper, we deal with twosided bounds for so-called $p$-Schwab-Borchardt mean and four special means generated by this one-parameter mean. All these means have been introduced and investigated in our recent paper [1]. A special case of the $p$-Schwab-Borchardt mean is discussed in the author's earlier papers [2-4]. This work, which is continuation of [1], is organized as follows. Definitions of generalized trigonometric and hyperbolic functions are included in Section 2. Main results of this paper are established in Section 3.

\section{Definitions and Preliminaries}

For the reader's convenience, we recall first definition of the celebrated Gauss hypergeometric function $F(\alpha, \beta ; \gamma ; z)$ :

$$
F(\alpha, \beta ; \gamma ; z)=\sum_{n=0}^{\infty} \frac{(\alpha, n)(\beta, n)}{(\gamma, n)} \frac{z^{n}}{n !}, \quad|z|<1,
$$

where $(\alpha, n)=\alpha(\alpha+1) \cdots(\alpha+n-1)(n \neq 0)$ is the shifted factorial or the Appell symbol, with $(\alpha, 0)=1$ if $\alpha \neq 0$ and $\gamma \neq 0,-1,-2, \ldots$ (see, e.g., [5]).

In what follows, we will assume that the parameter $p$ is strictly greater than 1 . In some cases, this assumption will be relaxed to $1<p \leq 2$. We will adopt notation and definitions used in [6]. Let

$$
\pi_{p}=2 \frac{\pi / p}{\sin (\pi / p)}
$$

Further, let

$$
a_{p}=\frac{\pi_{p}}{2}
$$

Also, let $I=(0,1)$. The generalized trigonometric and hyperbolic functions needed in this paper are the following homeomorphisms:

$$
\begin{aligned}
\sin _{p}:\left(0, a_{p}\right) \longrightarrow I, & \cos _{p}:\left(0, a_{p}\right) \longrightarrow I, \\
\sinh _{p}:(0, \infty) \longrightarrow(0, \infty), & \cosh _{p}:(0, \infty) \longrightarrow(1, \infty) .
\end{aligned}
$$

The inverse functions $\sin _{p}^{-1}$ and $\sinh _{p}^{-1}$ are represented as follows [7]:

$$
\begin{aligned}
\sin _{p}^{-1} u & =\int_{0}^{u}\left(1-t^{p}\right)^{-1 / p} d t=u F\left(\frac{1}{p}, \frac{1}{p} ; 1+\frac{1}{p} ; u^{p}\right) \\
\sinh _{p}^{-1} u & =\int_{0}^{u}\left(1+t^{p}\right)^{-1 / p} d t=u F\left(\frac{1}{p}, \frac{1}{p} ; 1+\frac{1}{p} ;-u^{p}\right) .
\end{aligned}
$$


Inverse functions of the remaining two functions can be expressed in terms of $\sin _{p}^{-1}$ and $\sinh _{p}^{-1}$. We have

$$
\begin{aligned}
\cos _{p}^{-1} u & =\sin _{p}^{-1}\left(\sqrt[p]{1-u^{p}}\right) \\
\cosh _{p}^{-1} u & =\sinh _{p}^{-1}\left(\sqrt[p]{u^{p}-1}\right) .
\end{aligned}
$$

Generalized trigonometric functions recently have attracted attention of several researchers. The interested reader is referred to a highly cited paper by Lindqvist [8] and other papers (see, e.g., [9-12]) as well.

For the later use, we recall now definition of a certain bivariate mean introduced recently in [1]

$$
\mathrm{SB}_{p}(x, y)= \begin{cases}\frac{\sqrt[p]{y^{p}-x^{p}}}{\cos _{p}^{-1}(x / y)}, & 0 \leq x<y \\ \frac{\sqrt[p]{x^{p}-y^{p}}}{\cosh _{p}^{-1}(x / y)}, & y<x \\ x, & x=y .\end{cases}
$$

And we call $\mathrm{SB}_{p}(x, y)$ the $p$-Schwab-Borchardt mean. When $p=2$, the latter mean becomes a classical Schwab-Borchardt mean which has been studied extensively in mathematical literature (see, e.g., $[2-4,13,14])$. It is clear that $\mathrm{SB}_{p}(x, y)$ is a nonsymmetric and homogeneous function of degree 1 of its variables.

A remarkable result states that the mean $\mathrm{SB}_{p}$ admits a representation in terms of the Gauss hypergeometric function [1]:

$$
\mathrm{SB}_{p}(x, y)=y\left[F\left(\frac{1}{p}, \frac{1}{p} ; 1+\frac{1}{p} ; 1-\left(\frac{x}{y}\right)^{p}\right)\right]^{-1} .
$$

We close this section with definitions of some bivariate means used in the sequel. To this end, we will assume that $x, y>0$. The power mean $A_{r}(x, y)(r \in \mathbb{R})$ of order $r$ of $x$ and $y$ is defined in a usual way as

$$
A_{r}(x, y) \equiv A_{r}= \begin{cases}\sqrt[r]{\frac{x^{r}+y^{r}}{2}}, & r \neq 0 \\ \sqrt{x y}, & r=0 .\end{cases}
$$

The power mean $A_{0}$ is usually denoted by $G$. It is well known that the power mean $A_{r}$ is a strictly increasing function of $r$.

We will deal with a quadruple of bivariate means of $x$ and $y$. They are denoted by $L_{p}, P_{p}, M_{p}$, and $T_{p}(p>1)$, they have been introduced in [1], and they are defined as follows:

$$
\begin{gathered}
L_{p}(x, y) \equiv L_{p}=\operatorname{SB}_{p}\left(A_{p / 2}, G\right), \\
P_{p}(x, y) \equiv P_{p}=\operatorname{SB}_{p}\left(G, A_{p / 2}\right), \\
T_{p}(x, y) \equiv T_{p}=\operatorname{SB}_{p}\left(A_{p / 2}, A_{p}\right), \\
M_{p}(x, y) \equiv M_{p}=\operatorname{SB}_{p}\left(A_{p}, A_{p / 2}\right) .
\end{gathered}
$$

In the case when $p=2$, these means become the classical logarithmic mean $L$, two Seiffert means $P$ and $T$ (see $[15,16]$ ), and the Neuman-Sándor mean $M$ introduced in [3].

It is worth mentioning that the means defined above satisfy the chain of inequalities [1]:

$$
G<L_{p}<P_{p}<A_{p / 2}<M_{p}<T_{p}<A_{p}
$$

\section{Main Results}

First, we will deduce lower and upper bounds for the mean $\mathrm{SB}_{p}$. They have form of the weighted geometric and arithmetic means of $x$ and $y$.

Throughout the sequel, we will always assume that the parameter $p$ satisfies $p>1$ unless otherwise stated. Also, let

$$
\gamma_{p}=\frac{1}{p+1} .
$$

We will prove now the following.

Proposition 1. Let $x$ and $y$ be positive and unequal numbers. If $x<y$, then the two-sided inequality

$$
x^{\gamma_{p}} y^{1-\gamma_{p}}<\operatorname{SB}_{p}(x, y)<\gamma_{p} x+\left(1-\gamma_{p}\right) y
$$

holds true, where the second inequality in (17) is valid only if $1<p \leq 2$. Otherwise, if $x>y$, then

$$
x^{\gamma_{p}} y^{1-\gamma_{p}}<\mathrm{SB}_{p}(x, y)<\beta_{p} x+\left(1-\beta_{p}\right) y,
$$

where

$$
\beta_{p}= \begin{cases}\gamma_{p} & 1<p \leq 2 \\ \frac{1}{3} & p \geq 2\end{cases}
$$

Proof. Suppose that $x<y$. Using the first part of (8), we get

$$
\mathrm{SB}_{p}(x, y)=y \frac{\sqrt[p]{1-(x / y)^{p}}}{\cos _{p}^{-1}(x / y)}
$$

Letting

$$
\frac{x}{y}=\cos _{p} t
$$

and next applying the formula

$$
\sin _{p} t=\sqrt[p]{1-\left(\cos _{p} t\right)^{p}}
$$

(see, e.g., [6] or (6)), we obtain

$$
\mathrm{SB}_{p}(x, y)=y \frac{\sin _{p} t}{t} .
$$

The lower bound for $\mathrm{SB}_{p}(x, y)$ is the weighted geometric mean of $x$ and $y$; that is,

$$
x^{\alpha} y^{1-\alpha}<\mathrm{SB}_{p}(x, y) \text {. }
$$


Applying (21) to the left side of (24) and (23) to the right side of the last inequality yields

$$
\left(\cos _{p} t\right)^{\alpha}<\frac{\sin _{p} t}{t} .
$$

We appeal now to Theorem 3.6 in [7] to conclude that the last inequality is satisfied for all $\alpha \in\left(0, a_{p}\right]$ with optimal value $\alpha=$ $\gamma_{p}$. The upper bound for $\mathrm{SB}_{p}(x, y)$ is a weighted arithmetic mean of $x$ and $y$; that is,

$$
\mathrm{SB}_{p}(x, y)<\beta x+(1-\beta) y .
$$

Using (21) and (23), we can write the last inequality as follows:

$$
\frac{\mathrm{SB}_{p}(x, y)}{y}<\beta \cos _{p} t+1-\beta
$$

Utilizing inequality (3.23) in [7]

$$
\frac{\sin _{p} t}{t}<\frac{\cos _{p} t+p}{p+1}
$$

$(1<p \leq 2)$ and (23), we obtain the desired result. We will establish now bounds (18) which are valid provided that $x>y$ and $p>1$. To this aim, we use a second part of (8) to obtain

$$
\mathrm{SB}_{p}(x, y)=y \frac{\sqrt[p]{(x / y)^{p}-1}}{\cosh ^{-1}(x / y)} .
$$

Letting $x / y=\cosh _{p} t$ and taking into account that $\sqrt[p]{\left(\cosh _{p} t\right)^{p}-1}=\sinh _{p} t($ see $(7))$, we obtain

$$
\frac{\mathrm{SB}_{p}(x, y)}{y}=\frac{\sinh _{p} t}{t} .
$$

The lower bound for $\mathrm{SB}_{p}(x, y)$ is

$$
x^{\alpha} y^{1-\alpha}<\mathrm{SB}_{p}(x, y) .
$$

Taking into account that $x / y=\cosh _{p} t(t>0)$, inequality (31) can be written as

$$
\left(\cosh _{p} t\right)^{\alpha}<\frac{\sinh _{p} t}{t} .
$$

It has been demonstrated in [7, Theorem 3.8] that the last inequality is satisfied for all $\alpha \in\left(0, \gamma_{p}\right]$ with an optimal value if $\alpha=\gamma_{p}$. This completes the proof of the left inequality in (18). To obtain the right-hand side inequality in (18), we employ the following inequalities:

$$
\frac{\sinh _{p} t}{t}<\gamma_{p} \cosh _{p} t+1-\gamma_{p}
$$

if $1<p \leq 2$ and

$$
\frac{\sinh _{p} t}{t}<\frac{\cosh _{p} t+2}{3}
$$

if $p \geq 2$. Formula (19) now follows and the proof is complete. [3].

Bounds (17) and (18), when $p=2$, have been obtained in

We will apply now Proposition 1 to obtain two-sided bounds for the four bivariate means $P_{p}, T_{p}, L_{p}$, and $M_{p}$ defined in Section 2 . The lower bounds are weighted geometric means of either $\left(G, A_{p / 2}\right)$ or $\left(A_{p / 2}, A_{p}\right)$. The upper bound is the weighted arithmetic means of the same pairs of elementary bivariate means.

We have the following.

Proposition 2. Let the numbers $\gamma_{p}$ and $\beta_{p}$ be the same as in (16) and (19). Then,

$$
\begin{gathered}
G^{\gamma_{p}} A_{p / 2}^{1-\gamma_{p}}<P_{p}<\gamma_{p} G+\left(1-\gamma_{p}\right) A_{p / 2}, \\
A_{p / 2}^{\gamma_{p}} A_{p}^{1-\gamma_{p}}<T_{p}<\gamma_{p} A_{p / 2}+\left(1-\gamma_{p}\right) A_{p},
\end{gathered}
$$

where the right inequalities in (35) and (36) hold true provided that $1<p \leq 2$. Also,

$$
\begin{aligned}
& A_{p / 2}^{\gamma_{p}} G^{1-\gamma_{p}}<L_{p}<\gamma_{p} A_{p / 2}+\left(1-\gamma_{p}\right) G, \\
& A_{p}^{\gamma_{p}} A_{p / 2}^{1-\gamma_{p}}<M_{p}<\gamma_{p} A_{p}+\left(1-\gamma_{p}\right) A_{p / 2} .
\end{aligned}
$$

Proof. In order to establish inequality (35), we use (12) and next apply (17) with $x:=G$ and $y:=A_{p / 2}$. The remaining inequalities (36)-(37) can be proven in an analogous manner. We omit further details.

\section{Conflict of Interests}

The author declares that there is no conflict of interests regarding publication of this paper.

\section{References}

[1] E. Neuman, "On the p-version of the Schwab-Borchardt mean," International Journal of Mathematics and Mathematical Sciences, vol. 2014, Article ID 697643, 7 pages, 2014.

[2] E. Neuman, "Inequalities for the Schwab-Borchardt mean and their applications," Journal of Mathematical Inequalities, vol. 5, no. 4, pp. 601-609, 2011.

[3] E. Neuman and J. Sándor, "On the Schwab-Borchardt mean," Mathematica Pannonica, vol. 14, no. 2, pp. 253-266, 2003.

[4] E. Neuman and J. Sándor, "On the Schwab-Borchardt mean II," Mathematica Pannonica, vol. 17, no. 1, pp. 49-59, 2006.

[5] B. C. Carlson, Special Functions of Applied Mathematics, Academic Press, New York, NY, USA, 1977.

[6] B. A. Bhayo and M. Vuorinen, "Inequalities for eigenfunctions of the p-Laplacian," Issues of Analysis, vol. 2(20), no. 1, pp. 13-35, 2013.

[7] R. Klén, M. Vuorinen, and X. Zhang, "Inequalities for the generalized trigonometric and hyperbolic functions," Journal of Mathematical Analysis and Applications, vol. 409, no. 1, pp. 521529, 2014

[8] P. Lindqvist, "Some remarkable sine and cosine functions," Ricerche di Matematica, vol. 44, no. 2, pp. 269-290, 1995.

[9] E. Neuman, "On the inequalities for the generalized trigonometric functions," International Journal of Analysis, vol. 2014, Article ID 319837, 5 pages, 2014. 
[10] E. Neuman, "Inequalities involving generalized trigonometric and generalized hyperbolic functions," Journal of Mathematical Inequalities, vol. 8, no. 4, pp. 725-736, 2014.

[11] E. Neuman, "Inequalities for the generalized trigonometric, hyperbolic and Jacobian elliptic functions," Journal of Mathematical Inequalities. In press.

[12] E. Neuman, "Wilker and Huygens-Type inequalities for the generalized trigonometric and for the generalized hyperbolic functions," Applied Mathematics and Computation, vol. 230, pp. 211-217, 2014.

[13] B. C. Berndt, J. M. Borwein, and P. B. Borwein, Pi and the AGM-A Study in Analytic Number Theory and Computational Complexity, Wiley, New York, NY, USA, 1987.

[14] B. C. Carlson, "Algorithms involving arithmetic and geometric means," The American Mathematical Monthly, vol. 78, no. 5, pp. 496-505, 1971.

[15] H.-J. Seiffert, "Problem 887," Nieuw Archief voor Wiskunde, vol. 11, p. 176, 1993.

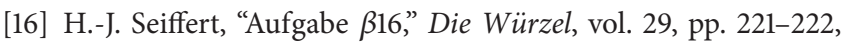
1995. 


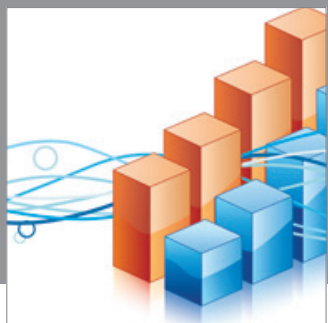

Advances in

Operations Research

mansans

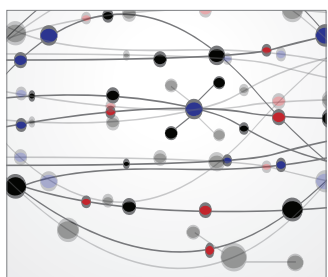

The Scientific World Journal
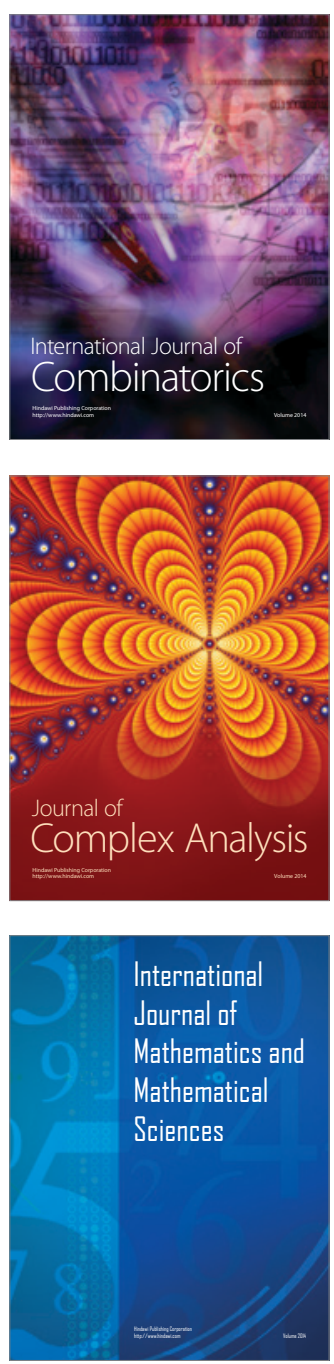
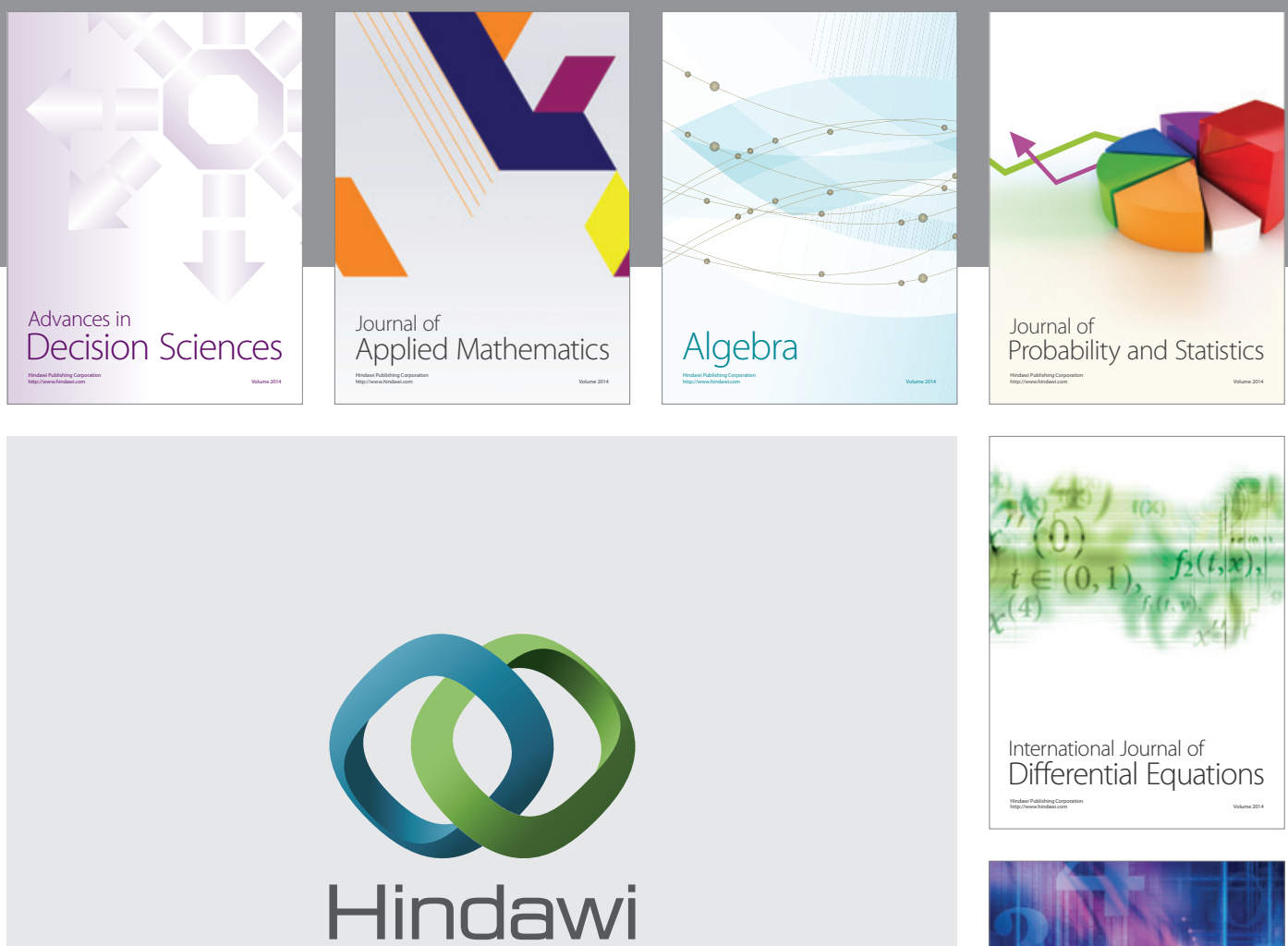

Submit your manuscripts at http://www.hindawi.com
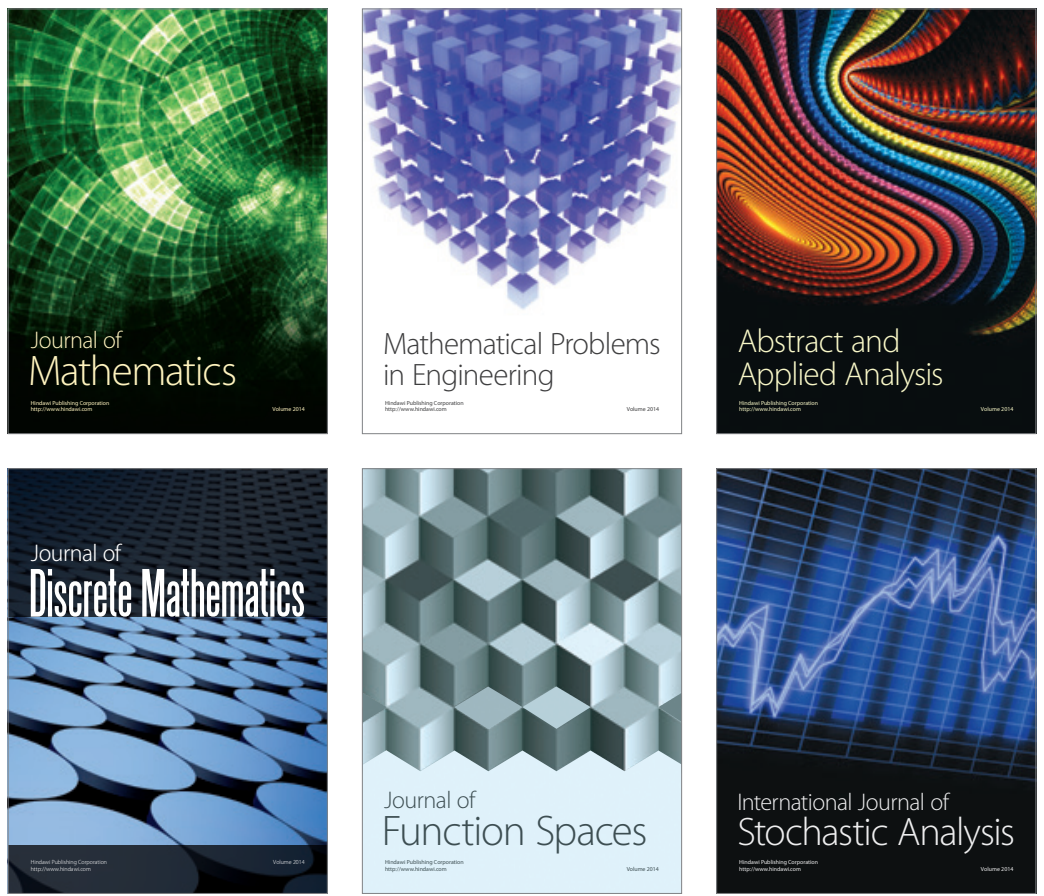

Journal of

Function Spaces

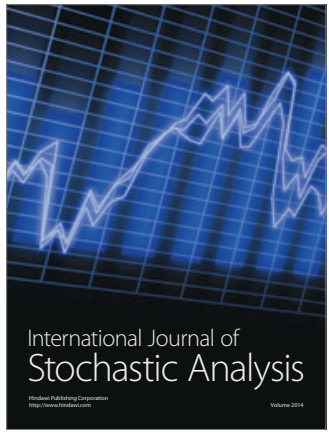

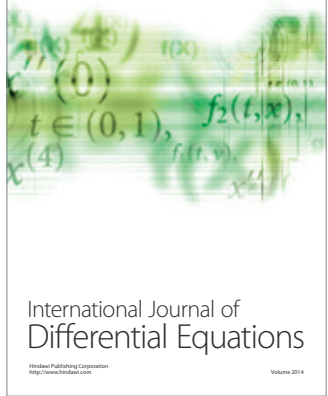
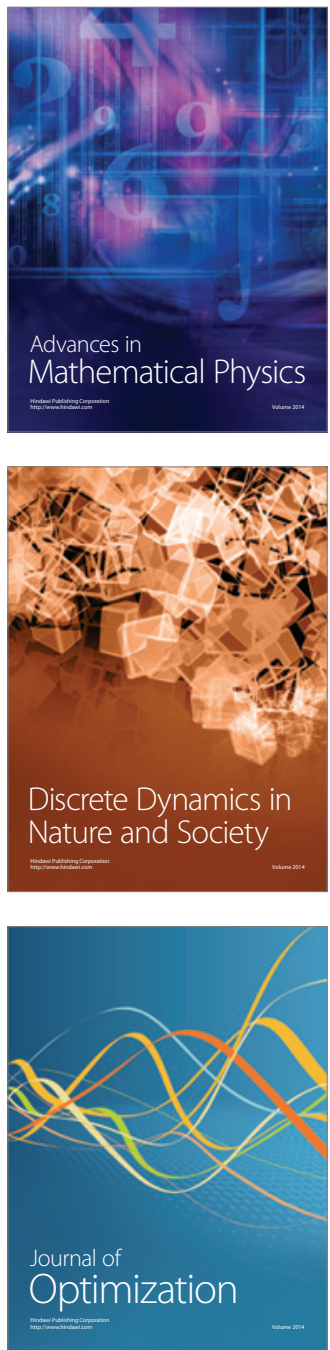